\title{
Frequency and severity of OSAHS in Mexican adolescents. The role of BMI and neck circumference
}

\author{
Armando Partida-Gaytán ${ }^{1}$, Arturo Berber ${ }^{1}$, Blanca Estela del Río-Navarro ${ }^{1^{*}}$, \\ Reyes Haro-Valencia ${ }^{2}$ \\ ${ }^{1}$ Departamento de Alergia e Inmunología Clínica Pediátrica, Hospital Infantil de México Federico Gómez, Instituto Nacional de \\ Salud, Secretaria de Salud, Distrito Federal, México; ${ }^{*}$ Corresponding Author: blancadelrionavarro@gmail.com \\ ${ }^{2}$ Chief of Sleep Breathing Disorders Clinic, Universidad Nacional Autónoma de México, Hospital General de México, \\ Secretaria de Salud, Distrito Federal, México
}

Received 18 June 2013; revised 14 July 2013; accepted 24 July 2013

Copyright (C) 2013 Armando Partida-Gaytán et al. This is an open access article distributed under the Creative Commons Attribution License, which permits unrestricted use, distribution, and reproduction in any medium, provided the original work is properly cited.

\section{ABSTRACT}

Background: Overweight and obesity are major public-health issues in Mexico. Sleep breathing disorders directly impact the health of affected subjects. The aim of this study was to describe the frequency and severity of obstructive sleep apnea/hypopnea syndrome (OSAHS) in Mexican adolescents grouped by weight status. Secondary objectives were to describe the influence of body mass index (BMI) and neck circumference (NC) as predictive factors of OSAH. Method: Somatometric, demographic data and full-night polysomnography were done. Linear and logistic regression models described the relationship between BMI and NC with OSAHS presence and severity. Receiver-Operating-Characteristics (ROC) curves showed different sensitivity and specificity performances of BMI and NC cutoff-values. Results: OSAHS frequency and severity were significantly greater in obese and extremelyobese adolescents (8\% vs $22 \%$ vs $67 \%$; $p<$ 0.001). Linear regression showed an $\mathrm{AHI}$ increase of 0.940 events/hour for every centimeter-increase in NC and 0.696 events/hour for every $1 \mathrm{~kg} / \mathrm{m}^{2}$-increase in BMI. Logistic-regression showed extreme-obesity as the main risk factor (OR 9.9) for diagnosis of OSAHS. ROC curves showed an area under the curve (AUC) of 0.83 for BMI defined cutoff-values, and of 0.80 for BMI percentile-for-age cutoff-values. The AUC of NC ROC curve was $\mathbf{0 . 8 0 4 3 . ~ C o n c l u s i o n : ~}$ This is the largest study made on Latin-American population evaluating the frequency and severity of OSAHS with full-night polysomnography. We showed that obese and extremely- obese Mexican-adolescents are at highest risk for OSAHS. Increasing BMI and NC showed positive correlation with AHI. Different BMI cut-off values showed similar diagnostic performance. Extreme-obesity increases 8 times the risk association for OSAHS.

Keywords: Obesity; Obstructive Sleep Apnea Hyopopnea Syndrome (OSAHS); Obstructive Sleep Apnea; Sleep Apnea Syndromes; Polysomnography; Body Mass Index (BMI); Neck Circumference; Adolescents

\section{INTRODUCTION}

National epidemiological data in Mexico, establish adolescents (10 to 19 years) as the most numerous group of the country's population pyramid. In the last four decades, overweight and obesity have been increasingly affecting Mexican population, every year affecting younger groups. According to the last National Nutrition and Health Survey data (Encuesta Nacional de Salud y Nutrición; ENSANUT 2012), 9.7\% of toddlers, $34.4 \%$ of scholars, $35 \%$ teenagers and $71 \%$ of adults live with overweight or obesity [1].

Medical literature has consistently evidenced the health risks associated to these malnutrition conditions. Among the most frequently reported, conditions are orthopedic, respiratory, sleep, metabolic and cardiovascular disorders, as well as premature death [2-4]. Abreast with these comorbidities, ENSANUT data estimated that direct and indirect costs have at least quadruplicated in the last 30 years, making overweight and obesity major public health concerns in México [1].

Sleep breathing disorders (i.e. primary snoring, hypopnea syndrome, central hypoventilation syndrome and 
obstructive sleep apnea/hypopnea syndrome) are defined by the presence of resistance to the air-flow in the upper respiratory airways during sleep with interruption of the normal patterns of sleep [5]. With the exception of primary snoring, the sleep-breathing disorders are associated with increases in cellular oxidative stress, chronic subclinical inflammation, autonomic activation and loss of sleep homeostasis. Sleep breathing disorders are considered important risk factors for the development of cardiovascular diseases (i.e. systemic arterial hypertension, pulmonary arterial hypertension, cor pulmonale, and atherosclerosis), metabolic disorders (i.e. hyperleptinemia, insulin resistance, hypercholesterolemia, elevated transaminases, insulin-like growth factor type 1 (IGF-1), growth hormone $(\mathrm{GH})$, and insulin-like factor binding protein type 3 (IGFBP3) decrease) and neuro-cognitive disorders (depression, anxiety and attention deficit and hyperactivity disorder) $[6,7]$.

Latin American populations have shown increased susceptibility to some chronic non-transmissible diseases such as overweight, obesity, diabetes, metabolic syndrome, non-alcoholic fatty liver disease, arterial hypertension, ischemic heart disease and some cancers. Mexicans are importantly affected by cardiovascular and metabolic morbidity, in fact, these disorders are the main cause of hospitalization and mortality in Mexican adults $[8,9]$. According to ENSANUT 2012, the challenge of chronic non-transmissible diseases starts at young age, for instance, in Mexican adolescents from 12 to 16 years, $4 \%$ of the women and $2.2 \%$ of the men suffer from hypertension [1]. Population screening programs do not consider sleep breathing disorders and to the best of our knowledge. There is not a single published study that addresses sleep breathing disorders frequency and severity, nor the existing relationship between obesity and OSAHS in Latin American population.

The aim of this study was to determine and compare the frequency and severity of OSAHS in three specific groups of Mexican adolescents (i.e. eutrophic healthy, obese and extremely obese). Secondary objectives included the description of the sensitivity and specificity behavior of different BMI cutoff-values for the diagnosis of OSAHS and the description of the relationship between BMI and $\mathrm{NC}$ with the $\mathrm{AHI}$ as a surrogate of OSAHS severity. A risk analysis was also of interest to show the odds ratio (OR) for OSAHS according to BMI group.

\section{METHODS}

\subsection{Sample}

The study population consisted of three groups of adolescents from 11 to 18 years of age. Extremely-obese adolescents were parts of a cohort of patients seen in the
Bariatric Surgery Clinic of our institution and were consecutively recruited. The aother two groups (obese and healthy eutrophic adolescents paired for age and sex) were friends, family or invited volunteers known by the subjects in the first group, also consecutively recruited. Weight status was classified according to American Academy of Pediatrics (AAP) recommendations [3]. Exclusion criteria consisted of known lung, heart, endocrine, muscular or neurologic diseases, history of prematurity, treatment with systemic corticosteroids in the last month, adenoid or tonsils hypertrophy, current pregnancy or breastfeeding, and any dysmorphic syndrome. The study was approved by our institution's ethics, bio-security and investigation review boards.

\subsection{Protocol}

All subjects were first studied at our institution, somatometry and a complete clinical history were taken to make sure healthy individuals were so, and to document the existing known comorbidities of the subjects in the two other groups. Height, weight, neck, abdominal, waist circumference and vital signs were measured three times by two different clinicians and the mean value of these variables was registered. Two otorhinolaringologists assessed (independently and blinded) for significant adenoid or tonsils hypertrophy Grade III or IV (according to fiber-optic nasal endoscopy findings; defining adenoid or tonsils hypertrophy as $\geq 50 \%$ obstruction of the nose/ pharynx lumen). At a second programmed visit, a complete overnight ATS-based protocol polysomnography was undertook [10].

\subsection{Polysomnographic Variables}

The studied variables during overnight polysomnographic (PSG) registry consisted of vital signs (heart rate, electrocardiography (ECG), respiratory frequency and pulse-oximetry), respiratory variables (respiratory movements with chest and abdominal bands, detection of airflow from nose and mouth with oronasal thermistors, and effectiveness of respiration with pulse oximetry) facial and extremities muscles activity assessment (ocular, submental and tibial electromyogram), snoring registry (laryngeal microphone), sleep staging (10-20 systems electroencephalogram (EEG), electrooculogram (EOG), and electromyogram (EMG)), and audiovisual recording of sleep (infrared videotaping). The PSG registry was analyzed and interpreted by two polysomography technologists and one somnologist.

\subsection{Statistical Analyses}

Demographic and anthropometric data were evaluated by descriptive statistics. Proportions were compared with Mantel-Hanzel linear-by-linear association chi-squared 
test. Median comparisons by Kruskal-Wallis with a priori contrasts between healthy eutrophic adolescents as reference group versus obese and extremely obese adolescents. Also obese vs. extremely obese were applicable. A linear regression model was able to describe the relationship between BMI and neck circumference (NC) with the severity of OSAHS measured by the AHI. Risk analysis was made to determine de OR for OSAHS according to BMI group. All analyses were made on SPSS for Windows V 17.0.

\section{RESULTS}

A total of 136 adolescents were recruited, consisting of 49 healthy eutrophic adolescents (Group 1), 41 obese adolescents (Group 2) and 46 extremely obese adolescents (Group 3). A slight predominance of men was observed in the whole group 81 vs 55 , though no statistical differences between groups was observed (men in 57\%, $53 \%$, and $67 \% ;(p=0.416))$. Demographic and somatometric data, grouped by body mass index are shown in Table 1.

Total sleep-time and sleep-efficiency were significantly shorter for extremely-obese adolescents. The Apnea/Hipopnea Index (AHI), increased significantly in each ascending group (according to BMI; i.e. Group $1<$ Group $2<$ Group 3). The Arousal Index (AI) was significantly greater for extremely-obese adolescents. OSAHS frequency was significantly greater in obese and extremely-obese adolescents. Thirty one (67\%) of extremely-obese adolescents had OSAHS, compared to 4
(8\%) healthy eutrophic adolescents and $9(22 \%)$ obese adolescents. The four healthy eutrophic adolescents affected, had mild OSAHS. Seven obese and seventeen extremely-obese adolescents also had mild OSAHS. Two obese and seven extremely-obese adolescents had moderate OSAHS. Severe OSAHS was exclusively present in the extremely-obese adolescents. The rest of polysomnographic variables are analyzed in Table 2.

Linear regression analysis showed the relationship between NC and BMI with respect to AHI as depicted in Figures 1 and 2. For every centimeter increase of NC, the AHI increased 0.940 events/hour (95\% CI 0.637 to 1.243 ; $r 2=0.22 ; p \leq 0.001)$; and for each kilogram per squared meter increase of BMI, the AHI increased 0.696 events/ hour $(95 \%$ CI 0.477 to $0.906 ; r 2=0.233 ; p \leq 0.001)$. The $\beta$ coefficient of BMI increased to 1 (95\% CI 0.49 to 1.52 ; $p<0.05)$; when considering only subjects with $\mathrm{BMI} \geq 25$ $\mathrm{kg} / \mathrm{m}^{2}$, and to $0.97(95 \%$ CI 0.16 to $1.79 ; p<0.05)$ when analyzing only subjects with BMI $\geq 30 \mathrm{~kg} / \mathrm{m}^{2}$. Further analysis showed increased $\beta$ coefficients, though with no statistical significance [i.e. BMI $\geq 35 \mathrm{~kg} / \mathrm{m}^{2} \beta=0.98$ (95\% CI 0.35 to 2.32); $p=0.14 /$ and $\mathrm{BMI} \geq 40 \mathrm{~kg} / \mathrm{m}^{2} \beta=$ 1.5 (95\% CI 0.97 to 3.99$) ; p=0.214]$.

Evaluation for OSAHS presence by logistic regression analysis of age, sex, neck circumference, obesity and extreme obesity as independent variables showed extremeobesity as the main risk factor with an OR 9.9 (95\% CI 1.38 to 71.94 ; L.R. $X 2=45.63$; $p \leq 0.001)$. We further sub-analyzed the risk association of extreme-obesity and OSAHS-severity by ordered logistic regression, showing

Table 1. Demographic and anthropometric data of 136 adolescents grouped by BMI.

\begin{tabular}{|c|c|c|c|c|}
\hline$n=136$ & Eutrophic adolescents $n=49$ & Obese adolescents $n=41$ & Extremely-obese adolescents $n=46$ & $p$ \\
\hline \multicolumn{5}{|l|}{ Age (years) } \\
\hline (mean/median) & $13.4 / 14$ & $12.4 / 13$ & $13.7 / 14$ & \multirow{3}{*}{${ }^{*} p=0.002$} \\
\hline mean CI 95\% & $13-13.8$ & $12-12.8$ & $13.1-14.2$ & \\
\hline range & $11-16$ & $11-14$ & $11-14$ & \\
\hline (male/female) $n(\%)$ & $28(57 \%) / 21(43 \%)$ & $22(53 \%) / 19(47 \%)$ & $31(67 \%) / 15(33 \%)$ & \multirow[t]{2}{*}{ N.S. } \\
\hline $95 \%$ CI of $\%$ & $42 \%-71 \% / 28 \%-57 \%$ & $37 \%-69 \% / 30 \%-62 \%$ & $53 \%-81 \% / 19 \%-47 \%$ & \\
\hline \multicolumn{5}{|l|}{ Body Mass Index $\left(\mathrm{kg} / \mathrm{m}^{2}\right)$} \\
\hline (mean/median) & $19.5 / 19.4$ & $28.5 / 27.5$ & $38.6 / 37.4$ & \multirow{3}{*}{$p=0.002$} \\
\hline Mean $95 \% \mathrm{CI}$ & $18.9-20.1$ & $27.5-29.5$ & $37.2-40.2$ & \\
\hline range & $13.6-23.2$ & $22.6-34.4$ & $31.5-53.6$ & \\
\hline \multicolumn{5}{|l|}{ Neck circumference (cms) } \\
\hline (mean/median) & $31.3 / 30.5$ & $35.5 / 35.5$ & $43.8 / 44.0$ & \multirow{3}{*}{$p<0.001$} \\
\hline mean $95 \% \mathrm{CI}$ & $30.6-31.9$ & $34.6-36.1$ & $42.5-45.1$ & \\
\hline range & $27.9-36.8$ & $30.5-44$ & $35.0-54.5$ & \\
\hline
\end{tabular}

*Kruskal-Wallis differences of the median. 
Table 2. Polisomnographic data grouped by BMI.

\begin{tabular}{|c|c|c|c|c|}
\hline$n=136$ & Eutrophic adolescents $n=49$ & Obese adolescents $n=41$ & Extremely-obese adolescents $n=46$ & $p$ \\
\hline \multicolumn{5}{|c|}{ Apnea/Hypopnea Index (AHI) } \\
\hline events/hour (mean/median) & $1.52 / 0.70$ & $3.72 / 2.05$ & $14.98 / 7.1$ & \multirow{3}{*}{${ }^{+} p<0.001$} \\
\hline Mean/S.D. (range) & $2.55(0-13)$ & $5.3(0-27.8)$ & $18.13(0-68.6)$ & \\
\hline Mean $95 \% \mathrm{CI}$ & $0.78-2.28$ & $2.03-5.42$ & $9.60-20.37$ & \\
\hline \multicolumn{5}{|c|}{ Arousal Index (AI) } \\
\hline events/hour (mean/median) & $8.18 / 7.5$ & $9.81 / 7.45$ & $13.59 / 12$ & \multirow{3}{*}{${ }^{*} p<0.001$} \\
\hline Mean S.D. (range) & $5.58(1.9-28.7)$ & $10.1(0.5-67)$ & $8.4(1.4-39.3)$ & \\
\hline Mean $95 \% \mathrm{CI}$ & $6.54-9.82$ & $6.58-13$ & $11.1-16.1$ & \\
\hline \multicolumn{5}{|c|}{ Obstructive Sleep Apnea Syndrome } \\
\hline Absent $n / \%(95 \% \mathrm{CI})$ & $45 / 92 \%(83 \%-99 \%)$ & $32 / 78 \%(64 \%-91 \%)$ & $15 / 33 \%(18 \%-46 \%)$ & \multirow{2}{*}{${ }^{\$} p<0.001$} \\
\hline Present $n / \%(95 \% \mathrm{CI})$ & $4 / 8 \%(0.2 \%-16 \%)$ & $9 / 22 \%(9 \%-36 \%)$ & $31 / 67 \%(53 \%-81 \%)$ & \\
\hline Mild $n(\%)$ & $4(100 \%)$ & $7(78 \%)$ & $17(55 \%)$ & \multirow{3}{*}{${ }^{\&} p<0.001$} \\
\hline Moderate $n(\%)$ & 0 & $2(22 \%)$ & $7(22.5 \%)$ & \\
\hline Severe $n(\%)$ & 0 & 0 & $7(22.5 \%)$ & \\
\hline \multicolumn{5}{|c|}{ Total registry time (minutes) } \\
\hline Mean/S.D. & $477.35 / 20.73$ & $480.74 / 16.81$ & $490.32 / 21.98$ & \multirow{2}{*}{${ }^{\#} p<0.001$} \\
\hline Mean $95 \%$ CI & $471.27-438.44$ & $475.36-486.11$ & $483.79-496.85$ & \\
\hline \multicolumn{5}{|c|}{ Total sleep time (minutes) } \\
\hline Mean/S.D. & $424.20 / 31.45$ & $426.65 / 38.42$ & $404.79 / 47.70$ & \multirow{2}{*}{${ }^{++} p=0.032$} \\
\hline Mean $95 \%$ CI & $414.96-433.43$ & $414.36-438.94$ & $390.62-418.96$ & \\
\hline \multicolumn{5}{|c|}{ Stage I sleep time (minutes) } \\
\hline Mean / S.D. & $41.66 / 14.68$ & $41.81 / 17.14$ & $39.27 / 24.47$ & \multirow{2}{*}{ N.S. } \\
\hline Mean $95 \%$ CI & $31.34-45.97$ & $36.33-47.29$ & $32-46.53$ & \\
\hline \multicolumn{5}{|c|}{ Stage I sleep time (\% of total sleep time) } \\
\hline Mean/S.D. & $9.91 \% / 3.7 \%$ & $9.86 \% / 4 \%$ & $10.11 \% / 7.68 \%$ & \multirow{2}{*}{ N.S. } \\
\hline Mean $95 \%$ CI & $8.82 \%-10.99 \%$ & $8.58 \%-11.15 \%$ & $7.82 \%-12.39 \%$ & \\
\hline \multicolumn{5}{|c|}{ Stage II sleep time (minutes) } \\
\hline Mean/S.D. & $29.97 / 34.08$ & $211.73 / 31.4$ & $208.38 / 48.39$ & \multirow{2}{*}{${ }^{\$ \$} p=0.002$} \\
\hline Mean 95\% CI & $219.97-239.98$ & $201.69-221.78$ & $194.01-222.75$ & \\
\hline \multicolumn{5}{|c|}{ Stage II sleep time (\% of total sleep time) } \\
\hline Mean/S.D. & $54.09 \% / 6.11 \%$ & $49.53 \% / 4.87 \%$ & $50.85 \% / 8.06 \%$ & \multirow{2}{*}{${ }^{\$} p=0.002$} \\
\hline Mean $95 \%$ CI & $52.30 \%-55.89 \%$ & $47.97 \%-51.1 \%$ & $48.46 \%-53.25 \%$ & \\
\hline \multicolumn{5}{|c|}{ Stage III sleep time (minutes) } \\
\hline Mean/S.D. & $19.52 / 8.88$ & $25.83 / 26.01$ & $89.42 / 31.08$ & \multirow{2}{*}{$\stackrel{\&}{*} p<0.001$} \\
\hline Mean 95\% CI & $16.91-22.13$ & $17.51-34.15$ & $80.19-98.65$ & \\
\hline \multicolumn{5}{|c|}{ Stage III sleep time (\% of total sleep time) } \\
\hline Mean/S.D. & $4.61 \% / 2.10 \%$ & $6.18 \% / 6.75 \%$ & $22.12 \% / 7.97 \%$ & \multirow{2}{*}{${ }^{\&} p<0.001$} \\
\hline Mean $95 \%$ CI & $3.99 \%-5.22 \%$ & $4.02 \%-8.34 \%$ & $19.75 \%-24.48 \%$ & \\
\hline
\end{tabular}




\begin{tabular}{|c|c|c|c|c|}
\hline \multicolumn{5}{|c|}{ REM sleep time (minutes) } \\
\hline Mean/S.D. & $61.06 / 19.20$ & $67.61 / 17.45$ & $59.51 / 24.52$ & \multirow{2}{*}{ N.S. } \\
\hline Mean $95 \%$ CI & $55.42-66.70$ & $62.02-73.19$ & $52.22-66.79$ & \\
\hline \multicolumn{5}{|c|}{ REM sleep time (\% of total sleep time) } \\
\hline Mean/S.D. & $14.42 \% / 3.86 \%$ & $15.75 \% / 2.54 \%$ & $14.41 \% / 5.33 \%$ & \multirow{2}{*}{ N.S. } \\
\hline Mean $95 \%$ CI & $13.29 \%-15.56 \%$ & $14.62 \%-16.88 \%$ & $12.82 \%-15.99 \%$ & \\
\hline \multicolumn{5}{|c|}{ Oxygen Saturation } \\
\hline Mean/S.D. & $92.93 \% / 2.08 \%$ & $92.55 \% / 1.86 \%$ & $92.2 \% / 3.17 \%$ & \multirow{2}{*}{ N.S. } \\
\hline Mean $95 \%$ CI & $92.32 \%-93.55 \%$ & $91.95 \%-93.14 \%$ & $91.26 \%-93.14 \%$ & \\
\hline
\end{tabular}

${ }_{p}^{+} p<0.001$ for contrast 1 and $2 ;{ }^{++} p=0.137$ for contrast $1 ; p=0.025$ for contrast $2 ;{ }^{*} p<0.001$ for contrast $1 ; p=0.65$ for contrast $2 ;{ }^{* *} p=0.012$ for contrast $1 ; p$ $=0.003$ for contrast $2 ;{ }^{\$} p<0.001$ for contrast 1 and $2 ;{ }^{\$} \$ p=0.003$ for contrast $1 ; p=0.768$ for contrast $2 ;{ }^{*} p<0.001$ for contrast 1 and 2 ; \&\& $p<0.001$ for contrast 1 and $2 ;{ }^{\#} p=0.033$ for contrast $1 ; p=0.010$ for contrast 2; Comparissons between " $\mathrm{k}$ " independent samples, proportions compared by linear-by-linear $X^{2}$; means/medians compared by Kruskal-Wallis. Contrast 1 (eutrophic adolescents vs. obese adolescents and extremely-obese adolescents). Contrast 2 (obese adolescents vs. extremely-obese adolescents).

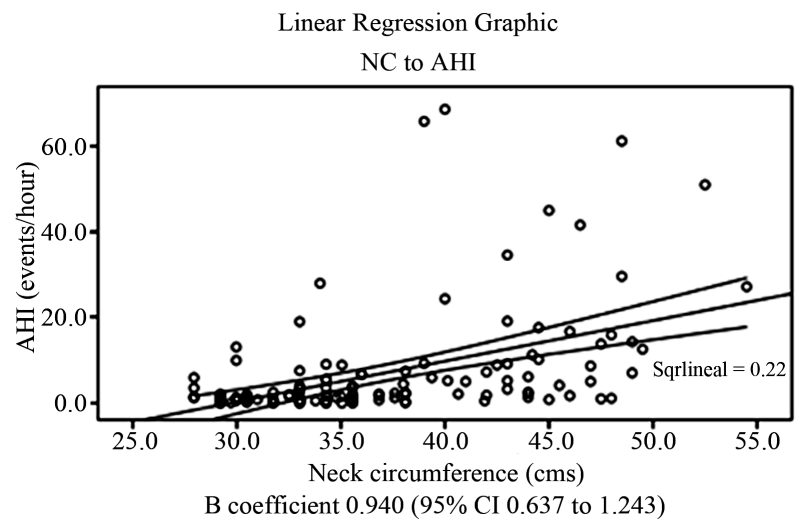

Figure 1. Linear relationship between NC and AHI.

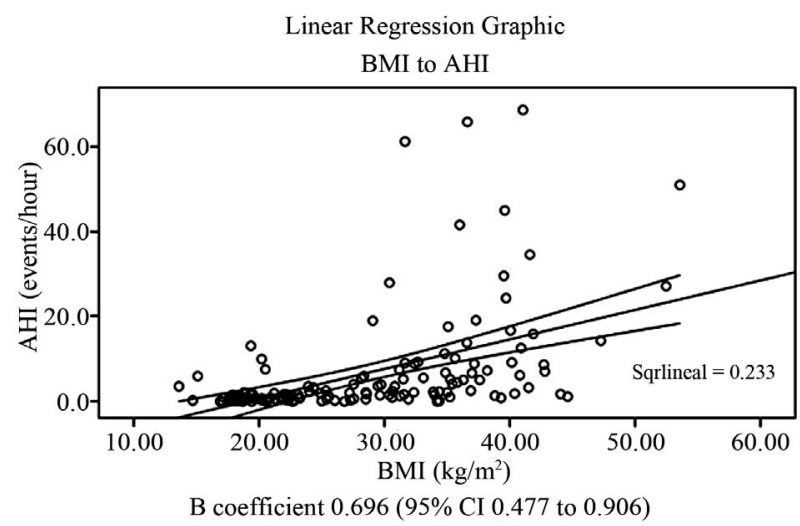

Figure 2. Linear relationship between BMI and AHI.

increased risk for all three grades of OSAHS (i.e. mild OSAHS $=$ OR $6.20,95 \%$ CI 1.75 - 10.65, moderate OSAHS $=$ OR 7.94, 95\% CI 3.37 - 12.51, and severe OSAHS $=$ OR $8.99,95 \%$ CI 4.34 - 13.63; L.R. $X 2=$ 49.96; $p \leq 0.001)$. The rest of the covariates are shown in Table 3.
ROC curve analysis allowed to compare the sensitivity and specificity of the two different criteria used to classify adolescents according to body mass index for the diagnosis of OSAHS. ROC curve for BMI values showed an area under the curve (AUC) of $0.8355(95 \%$ CI 0.765 - 0.895), while ROC curve for percentile values of BMI showed an AUC of 0.807 (95\% CI 0.732 - 0.871), showed in Figure 3. Specific values of sensitivity and specificity of pertinent used cutoff-points are showed in Table 4.

ROC curve analysis for neck circumference showed an AUC of 0.804 (95\% CI 0.724 - 0.854), shown in Figure 4. Specific values of sensitivity and specificity of the means of each group are showed in Table $\mathbf{5}$.

\section{DISCUSSION}

According to Guilleminault and coworkers, $10 \%$ of children with OSAHS are obese [11]. Marcus and coworkers described that $46 \%$ of non-selected obese children who underwent polysomnography resulted with OSAHS [12]. Silvestri and coworkers reported that 59\% of children referred to a sleep clinic and evaluated with polysomnography had OSAHS [13]. Kalra and coworkers described $55 \%$ of obese adolescents selected for bariatric surgery that underwent polysomnography had OSAHS [14]. Redline and coworkers made one of the few population-based studies which included 399 children between 2 - 18 years old, and found obesity as the principal risk factor for OSAHS with an odds ratio (OR) of 4.5 (95\% confidence interval 1.58 - 13.3) [15]. To our knowledge, no similar studies had been published in Latin American population to which we could compare our results. The findings in this study give evidence of an increased susceptibility for OSAHS in Mexican adolescents with the highest prevalence reported ever. In this 

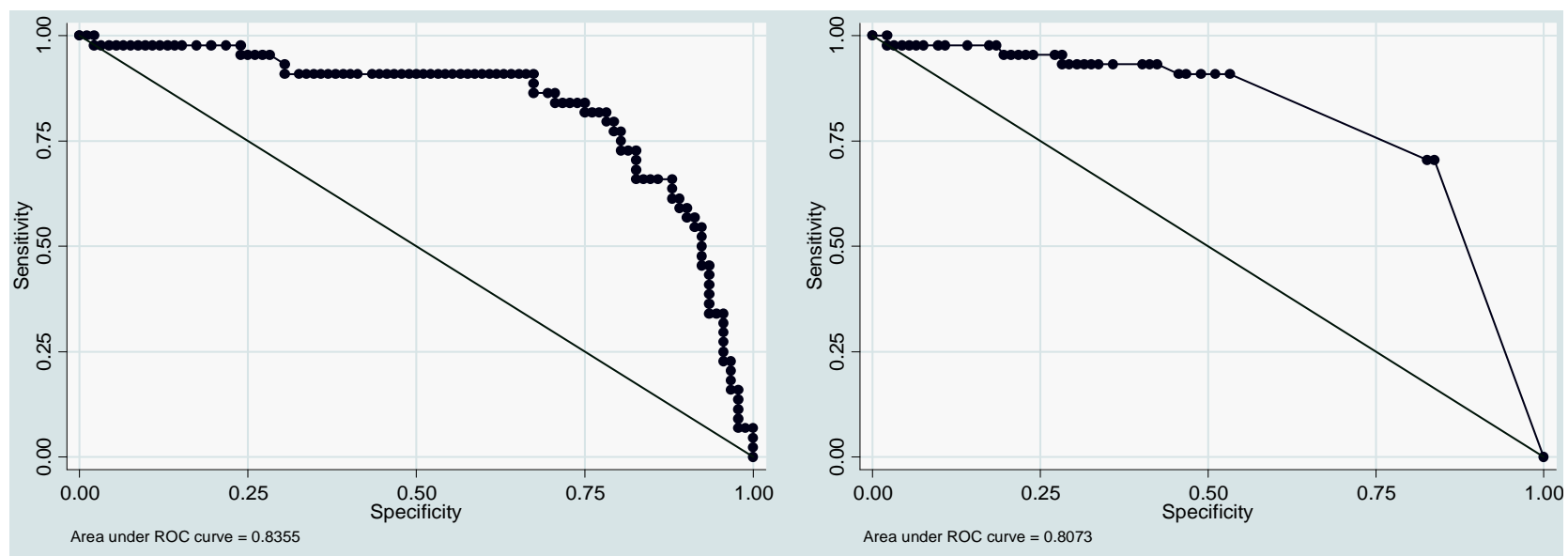

Figure 3. ROC curves for BMI cutoff-values (left) and BMI percentil cutoff-values (right).

Table 3. Polisomnographic data grouped by BMI.

\begin{tabular}{ccccc}
\hline Independent variable & Odds Ratio & $\boldsymbol{p}$ & \multicolumn{2}{c}{ [95\% Conf. Interval] } \\
\hline Age (years) & 1.150 & 0.399 & 0.832 & 1.589 \\
Neck circumference & 1.077 & 0.280 & 0.942 & 1.231 \\
Obesity & 2.714 & 0.190 & 0.609 & 12.088 \\
Extreme-obesity & 9.981 & 0.022 & 1.385 & 71.945 \\
Sex (male) & 1.253 & 0.628 & 0.502 & 3.128 \\
\hline
\end{tabular}

Table 4. Sensitivity and specificity values according to different BMI cutoff-points for the diagnosis of OSAHS ${ }^{+}$.

\begin{tabular}{ccccc}
\hline & Overweight & Obesity & Extreme/severe obesity \\
\hline BMI value & $\geq \mathbf{2 5} \mathbf{~ k g} / \mathbf{m}^{\mathbf{2}}$ & $\mathbf{2 3 0} \mathbf{~ k g} / \mathbf{m}^{\mathbf{2}}$ & $\mathbf{2 3 5} \mathbf{~ k g} / \mathbf{m}^{\mathbf{2}}$ & $\mathbf{2 4 0} \mathbf{~ k g} / \mathbf{m}^{\mathbf{2}}$ \\
\hline Sensitivity & $90 \%$ & $84 \%$ & $61 \%$ & $25 \%$ \\
Specificity & $56 \%$ & $74 \%$ & $89 \%$ & $96 \%$ \\
\hline $\begin{array}{c}\text { BMI percentile } \\
\text { for age (CDC) }\end{array}$ & $\geq \mathbf{8 5}$ & $\geq \mathbf{9 5}$ & $\geq \mathbf{9 9}$ \\
\hline $\begin{array}{c}\text { Sensitivity } \\
\text { Specificity }\end{array}$ & $90 \%$ & $90 \%$ & $70 \%$ \\
\hline
\end{tabular}

${ }^{+}$according to American Academy of Sleep Medicine; ${ }^{*}$ in some texts described as morbid obesity.

Table 5. Sensitivity and specificity values for the diagnosis of OSAHS $^{+}$according to the means of neck circumference of each group.

\begin{tabular}{cccc}
\hline & $\begin{array}{c}\text { Eutrophic } \\
\text { adolescents }\end{array}$ & $\begin{array}{c}\text { Obese } \\
\text { adolescents }\end{array}$ & $\begin{array}{c}\text { Extremely-obese } \\
\text { adolescents }\end{array}$ \\
\hline $\begin{array}{c}\text { Neck circumference } \\
\text { mean }\end{array}$ & $31.3 \mathrm{cms}$ & $35.5 \mathrm{cms}$ & $43.8 \mathrm{cms}$ \\
$\begin{array}{c}\text { Sensitivity } \\
\text { Specificity }\end{array}$ & $93 \%$ & $77 \%$ & $38 \%$ \\
\hline
\end{tabular}

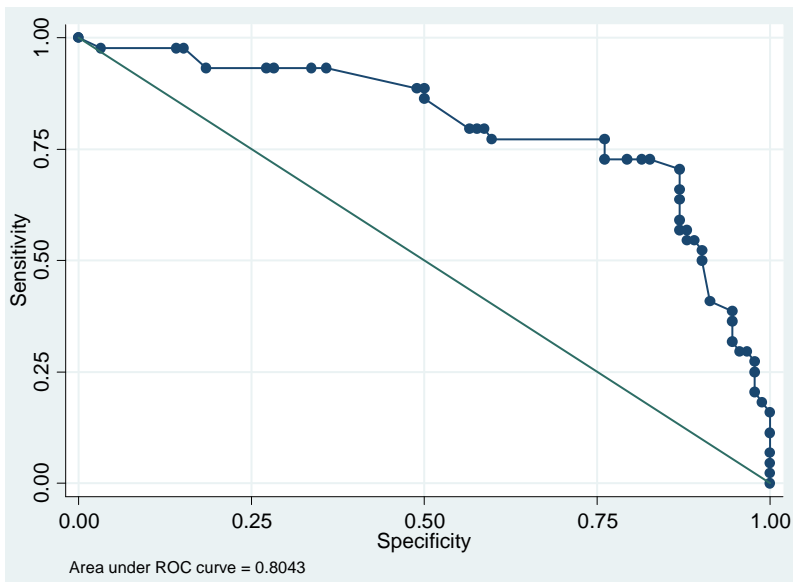

Figure 4. ROC curves for NC cutoff-values according to the means of each group.

study $90 \%$ of patients with OSAHS were obese or extremely-obese, with $22 \%$ of obese adolescents and $67 \%$ of extremely obese adolescents having OSAHS. These findings are important because according to national epidemiological data previously discussed (see introduction), Mexico has approximately $22 \%$ of its adolescents with overweight, $13 \%$ with obesity, representing the largest group in the population pyramid. To give an estimate of the burden of OSAHS in Mexican population, we calculated for the censed 22,000,000 adolescents, from which 2,860,000 estimated obese adolescents, and potentially 1,272,700 adolescents with OSAHS. Although the sample of our study was not meant to be representative of Mexican adolescent population, nor generalizable, the magnitude of the proportion-differences allowed to calculate the statistical power of the sample being of $94.19 \%$ for one-tailed and $90.27 \%$ for two-tailed with calculations comparing healthy eutrophic adolescents vs obese and extremely obese adolescents (i.e. $22 \%$ vs $44.5 \%$ ).

Many recent publications had described the clinical 
impact of OSAHS in health and disease. Inflammatory cascades are enhanced and perpetuated in patients with OSAHS mainly because of hypoxic-related stress, increased oxidative-stress, chronic subclinical inflammation, autonomic activation and sleep homeostasis-breakage. These different, though related, processes had been associated to clinical outcomes such as cardiovascular diseases (i.e. systemic arterial hypertension, pulmonary arterial hypertension, cor pulmonale, accelerated atherosclerosis), metabolic disorders (i.e. insulin resistance, hypercholesterolemia, transaminasemia, IGF-1, GH, IGFBP3 decrease, hyperleptinemia) and with neurocognitive disorders (i.e. depression, anxiety, attention deficit and hyperactivity disorder) among others. Because of the frequency and severity of OSAHS observed in this study, we strongly believe that OSAHS is an important diagnosis that should be looked-for in a more active manner such as glucose and hyperlipidemia screening programs. The cardiovascular and metabolic burden in Mexican adults is strongly manifest being the major causes of mortality in such population, early interventions oriented to diagnose, and consequently treat OSAHS, is highly likely to decrease cardiovascular and metabolic burden. As shown in this study, BMI and NC both could serve as easy-to-measure clinical variables capable of predicting OSAHS with a relatively good performance, as showed in the ROC curve of these variables, whether the clinician decides to use percentilar cutoff-values or specific BMI values, both having similar sensitivities and specificities. To the best of our knowledge, there is no public health policy statement in Mexico, Latin America or any other country approaching prompt diagnosis of OSAHS in overweight or obese patients. Clinical surrogates such as clinical questionnaires, overnight oximetry, audio recording, video recording and nap polysomnography (during daytime) have been reported to have poor negative predictive values. Because of the potential burden of OSAHS, there is an urgent need to develop specific strategies for earlier diagnosis and appropriate therapies. According to current obesity prevalence worldwide, it may be worthwhile, to approach this disease in a population-level basis.

LIMITATIONS: the current study did not explore previously validated questionnaires, nor the presence of any symptom associated to OSAHS, which could had been useful to analyze their different screening or diagnostic performances, when compared to gold-standard test (i.e. full-night polysomnography). We also did not include overweight subjects, which could potentially modify the dose-response effect seen between BMI, NC and AHI.

\section{CONCLUSION}

To our knowledge, this is the largest study made on
Latin American population evaluating the frequency and severity of OSAHS with full-night polysomnography. We showed that obese and extremely-obese Mexican adolescents are at higher risk for the development of OSAHS. Increasing BMI and NC showed positive correlation with AHI. Extremely-obese adolescents have the highest rate and severity of OSAHS. Sensitivity of BMI percentile cutoff-value was higher than conventional BMI cutoff-values used in adolescents, without a significant decrease on its specificity, showing similar diagnostic performances. Extreme-obesity increases 8 times the risk association for OSAHS.

\section{ACKNOWLEDGEMENTS}

We want to thank Alicia Castillo-Lazaro, Psychologist and Somnologist of Sleep Breathing Disorders Clinic, Universidad Nacional Autónoma de México at Hospital General de México Dr. Eduardo Liceaga together with their staff who made possible the performance and interpretation of polysomnographic studies. We also want to thank Dr. Carlos de la Torre, MD, Chief of Pediatric Otorhinolaryngology Department at Hospital Infantil de México Federico Gómez and his staff for the upper respiratory tract evaluation of many candidate subjects. Finally give thanks to Dr. Alexander Morales-Marin MD., Dr. Mario Pineda-Maldonado MD., who also participated in the recruitment and evaluation of study subjects.

\section{REFERENCES}

[1] Gutierrez, J.P., et al. (2012) Encuesta nacional de salud y nutrición 2012. Resultados Nacionales. Instituto Nacional de Salud Publica, Cuernavaca.

[2] Daniels, S.R. (2006) The consequences of childhood overweight and obesity. The Future of Children, 16, $47-$ 67. doi:10.1353/foc. 2006.0004

[3] Barlow, S.E., et al. (2007) Expert committee recommenddations regarding the prevention, assessment, and treatment of child and adoescent overweight and obesity: Summary report. Pediatrics, 120, 164-192. doi:10.1542/peds.2007-2329C

[4] Arens, R., et al. (2010) Childhood obesity and obstructive sleep apnea syndrome. Journal of Applied Physiology, 108, 436-444. doi:10.1152/japplphysiol.00689.2009

[5] Schechter, M.S., et al. (2002) Technical report: Diagnosis and management of childhood obstructive sleep apnea syndrome. Pediatrics, 109, 1-20. doi:10.1542/peds.109.4.e69

[6] Bhattacharjee, R., et al. (2011) Obesity and obstructive sleep apnea syndrome in children: A tale of inflammatory cascades. Pediatric Pulmonology, 46, 313-323. doi:10.1002/ppul.21370

[7] Katz, E.S., et al. (2010) Pediatric obstructive sleep apnea syndrome. Clinics in Chest Medicine, 31, 221-234. doi:10.1016/j.ccm.2010.02.002

[8] Barquera, S., et al. (2010) Obesidad en México, epidemiología y políticas de salud para su control y prevención. 
Gaceta Médica de México, 146, 389-396.

[9] Gracia, M., et al. (2010) La obesidad como enfermedad, la obesidad como problema social. Gaceta Médica de México, 146, 389-396.

[10] American Thoracic Society Official Statement. (1996) Standards and indications for cardiopulmonary sleep studies in children. American Journal of Respiratory and Critical Care Medicine, 153, 866-878.

[11] Guilleminault, C., et al. (2005) Pediatric obstructive sleep apnea syndrome. Archives of Pediatrics \& Adolescent Medicine, 159, 775-785. doi:10.1001/archpedi.159.8.775

[12] Marcus, C.L., et al. (1996) Obstructive sleep apnea in children. Seminars in Pediatric Neurology, 3, 23-28.
doi:10.1016/S1071-9091(96)80025-8

[13] Silvestri, J.M., et al. (1993) Polysomnography in obese cildren with history of sleep-associated breathing disorders. Pediatric Pulmonology, 16, 124-129. doi:10.1002/ppul.1950160208

[14] Kalra, M., Inge, T., Garcia, V., Daniels, S., Lawson, L., et al. (2005) Obstructive sleep apnea in extremely overweight adolescents undergoing bariatric surgery. Obesity Research, 13, 1175-1179. doi:10.1038/oby.2005.139

[15] Redline, S., et al. (1999) Risk factors for sleep-disordered breathing in children. American Journal of Respiratory and Critical Care Medicine, 159, 1527-1532. $\underline{\text { doi:10.1164/ajrccm.159.5.9809079 }}$ 